\title{
Chiral Enrichment of Serine via Formation, Dissociation, and Soft-Landing of Octameric Cluster Ions
}

\author{
Sergio C. Nanita, Zoltan Takats, and R. Graham Cooks \\ Department of Chemistry, Purdue University, West Lafayette, Indiana, USA
}

Sunnie Myung and David E. Clemmer

Department of Chemistry, Indiana University, Bloomington, Indiana, USA

\begin{abstract}
Chiral enrichment of serine is achieved in experiments that involve formation of serine octamers starting from non-racemic serine solutions. Serine octamers were generated by means of electrospray and sonic spray ionization of aqueous solutions of $d_{3}$-L-serine (108 Da) and D-serine (105 Da) having different molar ratios of enantiomers. A cyclic process involving the formation of chirally-enriched octameric cluster ions and their dissociation, viz. Ser $_{1} \rightarrow \mathrm{Ser}_{8}$ $\rightarrow$ Ser $_{1}$, allows serine monomers to be regenerated with increased enantiomeric excess as shown in two types of experiments: (1) Chiral enrichment in serine was observed in MS/MS/MS experiments in a quadrupole ion trap in which the entire distribution of serine octamers formed from non-racemic solutions was isolated, collisionally activated, and fragmented. Monomeric serine was regenerated with increased enantiomeric excess upon dissociation of octamers when compared with the enantiomeric composition of the original solution. (2) Chiral enrichment was observed in the products of soft-landing of mass-selected protonated serine octamers. These ions were generated by means of electrospray or sonic spray ionization, mass selected, and collected on a gold surface using ion soft-landing. Chiral enrichment of the soft-landed serine was established by redissolving the recovered material and comparing the intensities of protonated molecular ions of $d_{3}$-L-serine and D-serine after APCI-MS analysis. Both of these experiments showed comparable results, suggesting that formation of serine octamers depends only on the enantiomeric composition of the serine solution and that the magnitude of the chiral preference is intrinsic to octamers formed from solutions of given chiral composition. (J Am Soc Mass Spectrom 2004, 15, 1360-1365) (c) 2004 American Society for Mass Spectrometry
\end{abstract}

$\mathrm{W}$ e report chiral enrichment of serine through generation of octamers from non-racemic solutions in the absence of any other chiral reagent. These octameric clusters are formed enantiomerically-enriched when compared with the initial composition of the electrosprayed solution. Chiral enrichment of serine is achieved via cycles involving formation, isolation and dissociation of octamers (Ser $_{1}$ $\left.\rightarrow \mathrm{Ser}_{8} \rightarrow \mathrm{Ser}_{1}\right)$. In one particular experiment, the chirally-enriched product was recovered through softlanding of octamers on a gold surface.

The first reports that serine octamers are formed with a strong preference for homochirality [1-3] stimulated a series of studies that uncovered a number of unique features of the amino acid serine [4-12]. These

Published online August 4, 2004

Address reprint requests to Dr. R. G. Cooks, Department of Chemistry, Purdue University, 560 Oval Drive, West Lafayette, IN 47907, USA. E-mail: cooks@purdue.edu. studies support the hypothesis $[2,5]$ that serine octamers might have been involved in prebiotic mechanisms that lead to homochirogenesis. It has been demonstrated that protonated serine octamers undergo chiroselective reactions with compounds of fundamental importance to biochemistry, including other amino acids [5], glyceraldehyde [8], and glucose [8]. The chiral selection in these reactions occurs with preference for the enantiomers present in life (L-amino acids, Dsaccharides). Serine is also known to undergo chiral conversion under relatively mild conditions [8, 13, 14]. This is true for both the free amino acid [8] and for serine residues in proteins [13, 14]. The facile chiral conversion of serine might act [8] as a locus for symmetry breaking [15-20], the key problem in biochemical evolution.

In this paper, symmetry breaking is not dealt with but we demonstrate that chiral enrichment of serine can occur through octamer formation and dissociation. 
Chiral amplification has been extensively studied [21] in polymerization reactions, which involve cleavage and formation of covalent bonds. The chiral enhancement of serine described here involves fragile noncovalently bound aggregates in an open system (from which material may be lost). Chiral enrichment is proposed to involve enantioselective formation/dissociation of serine clusters, a process that may be related to the "majority rules" phenomenon observed in polymerization of homochiral monomers [22-24].

\section{Experimental}

The protonated octamer is generated by electrospray [25] or sonic spray ionization [26] and soft-landed [27, 28] on a gold surface using a preparative form of mass spectrometry. Dissociation of the octamers allows monomeric serine to be regenerated. This occurs with increased enantiomeric excess when compared with the composition of the original solution. The chiral consequences of interconversion between serine and its octamer have been studied by isotopically labeling one of the enantiomers. Isotopically labeled L-serine $\left(2,3,3-\mathrm{d}_{3}-\right.$ L-serine, $108 \mathrm{Da}$ ) was obtained from Cambridge Isotope Laboratories (Cambridge, MA), while D-serine was purchased from Sigma Co. (St. Louis, MO). Solutions were prepared in methanol/water/acetic acid (49:49:2 $\mathrm{vol} / \mathrm{vol})$. The total concentration of serine, $[\mathrm{Ser}]_{\mathrm{T}}=$ $\left[2,3,3-\mathrm{d}_{3}\right.$-L-Ser] + [D-Ser], was constant at $20 \mathrm{mM}$. The formation of chirally-enriched octamers has been demonstrated by single-stage MS experiments employing three different types of mass spectrometers. Chirallyenriched serine monomers were regenerated in two different sets of experiments, one of which allows the resulting material to be collected (soft-landing). The methods are summarized below.

\section{Single-Stage Mass Spectrometry}

Serine octamers were generated by electrospray ionization (ESI) or by sonic spray ionization (SSI). Detailed information about the home-built SSI source is available elsewhere [10]. Mass spectra were recorded and the chiral composition of octameric cluster ions was determined from the isotopic distribution. The experiments were performed using (1) a prototype Orbitrap mass spectrometer [29, 30], (2) a Finnigan LCQ Classic mass spectrometer (San Jose, CA), and (3) a home-built ion mobility/time-of-flight mass spectrometer [31].

\section{Tandem Mass Spectrometry}

The entire isotopic envelope of serine octamers $(\mathrm{m} / \mathrm{z}$ 841-865) was isolated, activated and dissociated in a Finnigan LCQ Classic mass spectrometer. Conditions were optimized such that the precursor ions (octamers) mainly yield monomers and dimers upon dissociation. The entire distribution of octamers was isolated in the ion trap (center mass $\mathrm{m} / \mathrm{z} 853$ and isolation window of $m / z$ 35). Dissociation of the selected precursor ions was subsequently achieved through collisional activation using helium buffer gas as the collision partner. The $\mathrm{MS}^{3}$ experiments were then completed by isolation, activation, and dissociation of the entire distribution of dimeric product ions $(\mathrm{m} / \mathrm{z} 211,214$, and 217; center mass $\mathrm{m} / \mathrm{z} 214$ and isolation window of $\mathrm{m} / \mathrm{z} 100$ ). The enantiomeric composition of the resulting monomeric serine was measured from the abundance of the ionic fragments $\left(2,3,3-\mathrm{d}_{3}-\mathrm{L}-\mathrm{Ser}+\mathrm{H}\right)^{+}$and $(\mathrm{D}-\mathrm{Ser}+\mathrm{H})^{+}$respectively.

\section{Soft-Landing of Serine Octamers}

A Finnigan SSQ-710C equipped with a single quadrupole mass filter and modified for ion soft-landing was used to isolate the entire isotopic distribution of protonated serine octamers $(\mathrm{m} / \mathrm{z}$ 841-865) and to soft land these ions at a gold surface. The product of soft-landing was analyzed by rinsing a spot (radius, $3 \mathrm{~mm}$ ) from the gold substrate using $10 \mu \mathrm{L}$ deionized water and infusing the rinse solution at $10 \mu \mathrm{L} / \mathrm{min}$ flow rate into an LCQ Classic mass spectrometer equipped with APCI source. Nebulizing gas flow rate was 60 units, auxiliary gas flow rate was 5 units, corona discharge current was $4 \mu \mathrm{A}$, source temperature was $250^{\circ} \mathrm{C}$, heated capillary temperature was $200{ }^{\circ} \mathrm{C}$. Spectra were collected in positive ion mode from 50 to $250 \mathrm{Th}$. Acquired profile spectra were averaged and ion intensities were integrated.

\section{Results and Discussion}

Solutions of serine of different enantiomeric composition were analyzed by means of electrospray ionization [25] mass spectrometry using a resolution of $\sim 10^{4}$ [29, 30]. As shown in Figure 1, a serine solution with small enantiomeric excess yielded octamers that are further enriched in the major optical isomer. The percent enantiomeric excess [(\% e.e.); absolute value of the difference between the molar fractions of the enantiomers expressed as a percentage of the maximum value] of serine in the octameric clusters was measured from the ESI mass spectra. For example, a serine solution with $20 \%$ e.e. yielded enantiomeric excesses in the octamers of $\sim 50 \%$ (Figure $1 \mathrm{c}$ and $\mathrm{f}$ ). This result demonstrates that formation of the octamers from non-racemic serine solution is accompanied by a significant increase in clusters of enriched chirality. For comparison, we also show mass spectra of the pure enantiomers (Figure 1a and $\mathrm{d}$ ) and the pure racemate (Figure 1g).

The fact that the serine octamers are enriched in the most abundant isomer allows the possibility of reforming the amino acid monomers with increased enantiomeric excess through cycles involving formation, isolation and dissociation of $\left(\mathrm{Ser}_{8}+\mathrm{H}\right)^{+}$ions. Attempts were made to regenerate chirally-enriched monomeric serine by this route. Collision-induced dissociation (CID) experiments were carried out and the chiral composition 


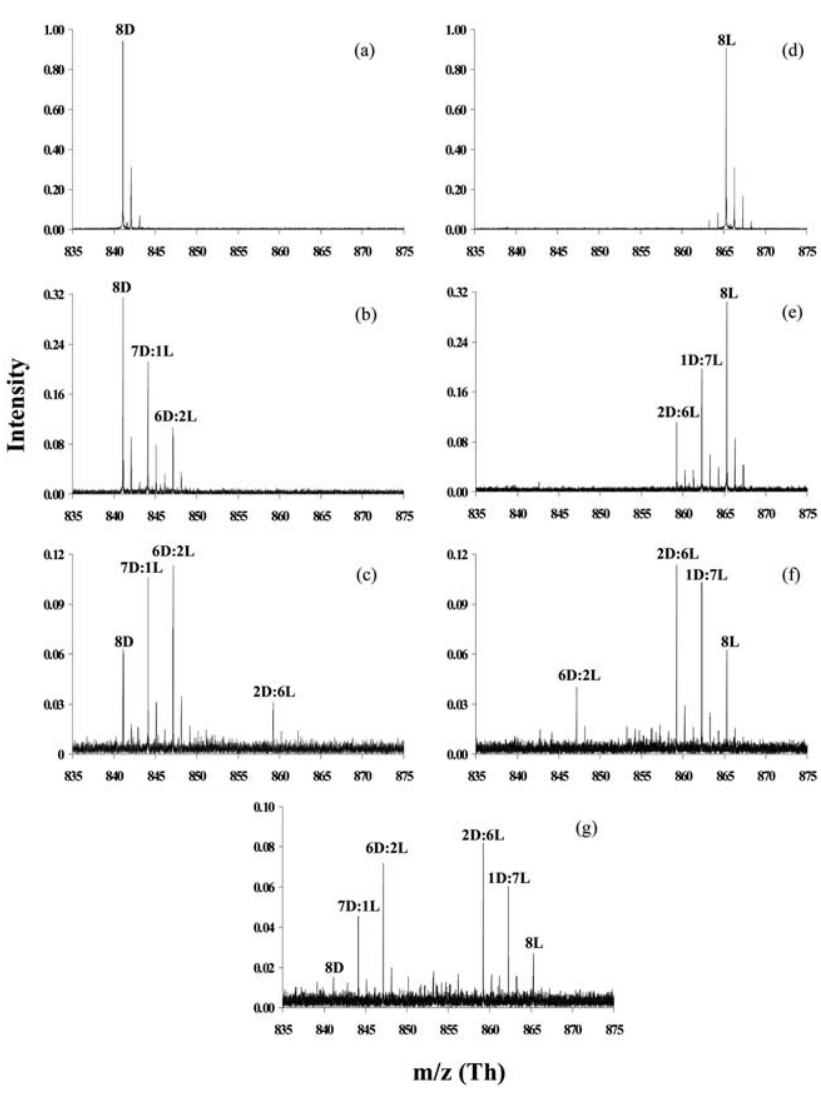

Figure 1. High-resolution isotope labeling mass spectra of serine mixtures (D-serine, $105 \mathrm{Da}$; 2,3,3- $\mathrm{d}_{3}$-L-serine, $108 \mathrm{Da}$ ) recorded using an Orbitrap mass spectrometer. The mass spectra correspond to solutions containing different enantiomeric compositions: (a) 100\% $\mathrm{D}, 0 \% \mathrm{~L}$; (b) $80 \% \mathrm{D}, 20 \% \mathrm{~L}$; (c) $60 \% \mathrm{D}, 40 \% \mathrm{~L}$; (d) $0 \% \mathrm{D}, 100 \% \mathrm{~L}$; (e) $20 \% \mathrm{D}, 80 \% \mathrm{~L}$; (f) $40 \% \mathrm{D}, 60 \% \mathrm{~L}$; (g) $50 \% \mathrm{D}, 50 \% \mathrm{~L}$.

of the resulting product ions was measured for solutions containing 2,3,3- $\mathrm{d}_{3}$-L-serine and D-serine in different proportions. Solutions containing one enantiomer in $20 \%$ e.e. gave a ratio of monomeric fragment ions of the octamers $(\mathrm{m} / \mathrm{z} 836$ to 870 isolated for CID) that corresponded to $(45 \pm 4) \%$ e.e. and $(48 \pm 5) \%$ e.e. for the $20 \%$ e.e. of $\mathrm{D}$ and $20 \%$ e.e. of $\mathrm{L}$ cases, respectively (Figure $2 \mathrm{a}$ and $b$ ). Note that in these experiments the entire distribution of octamers was isolated and activated by collision-induced dissociation and that this step generated protonated serine dimers. These dimers were then dissociated in a further CID step to form the singlyprotonated monomers whose relative abundances were measured. Three stages of mass analysis are needed since it is not possible to observe monomeric fragments from the octamer in a simpler $\mathrm{MS}^{2}$ experiment due to intrinsic fragment ion mass range limitations of the ion trap instrument.

In order to confirm that chiral enrichment of serine can be achieved through formation and dissociation of octamers, the ion soft-landing technique was employed [27, 28]. The use of ion soft-landing instead of destructive detection allows products derived from the octameric ions to be collected and subjected to

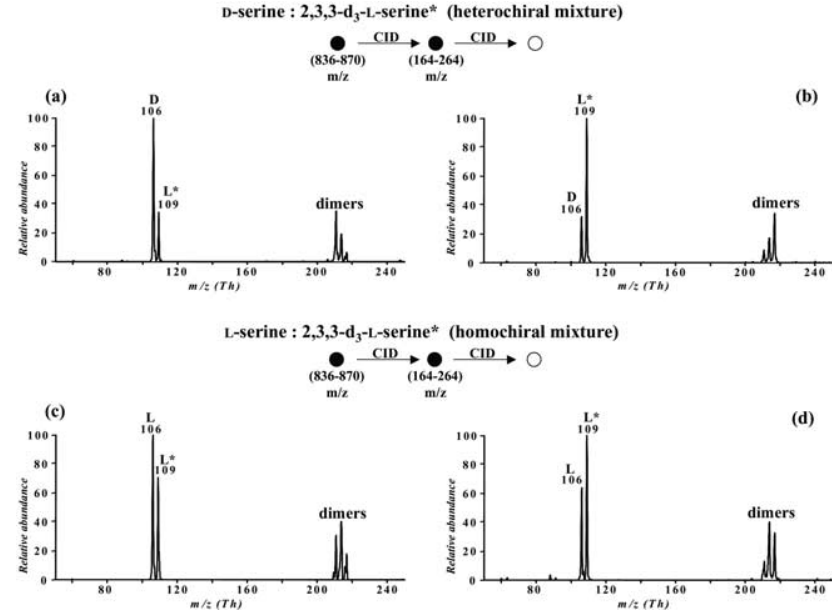

Figure 2. Product ion mass spectra of serine octamers. The octameric cluster ions were formed from heterochiral solutions containing: (a) 60\% D-serine, 40\% 2,3,3- $\mathrm{d}_{3}$-L-serine, (b) $40 \%$ Dserine, $60 \%$ 2,3,3- $\mathrm{d}_{3}-\mathrm{L}$-serine. The data confirm that serine octamers are chirally enriched in comparison to the starting non-racemic solution. Spectra (c) and (d) are $\mathrm{MS}^{\mathrm{n}}$ spectra recorded from homochiral mixtures of L-serine and 2,3,3- $\mathrm{d}_{3}$-L-serine $60: 40$ and 40:60 respectively, showing that homochiral octamers contain both types of L-serines in an statistical fashion, ruling out the possibility of isotope effects.

further analysis. This method of preparative mass spectrometry allows separation of the deposition of the serine octamer ions and the analysis of the landed sample. The analysis step can in principle be done using any suitable analytical technique, including alternative mass spectrometric methods such as atmospheric pressure chemical ionization (APCI) which was used here. The entire distribution of serine octamers formed from solutions containing $20 \%$ e.e. of the D- and L-isomer, respectively, was isolated and landed onto a gold surface. In the absence of an appropriate surface characterization technique, it is not possible to know whether the clusters remain intact or dissociate upon landing at the surface. Chirally-enriched serine was recovered from the surface after re-dissolving the collected material. Solutions of post-landing serine analyzed by APCI-MS yielded enantiomeric compositions of $(58 \pm 7) \%$ e.e. and $(54 \pm 8) \%$ e.e., respectively, in agreement with the gas-phase data (Figure 2).

The results just discussed have been produced using fundamentally different experiments on different instruments: an Orbitrap mass spectrometer [29] (data shown in Figure 1), a commercial Finnigan LCQ Classic quadrupole ion trap mass spectrometer (data shown in Figure 2), and a Finnigan SSQ-710C single quadrupole mass filter (modified for ion soft-landing). In addition, the data obtained from single-stage mass spectra (Figure 1) have been reproduced in a home-built hybrid ion mobility/time-of-flight mass spectrometer [31]. The similarity of the results strongly suggests that this behavior is intrinsic to the chemical system. 


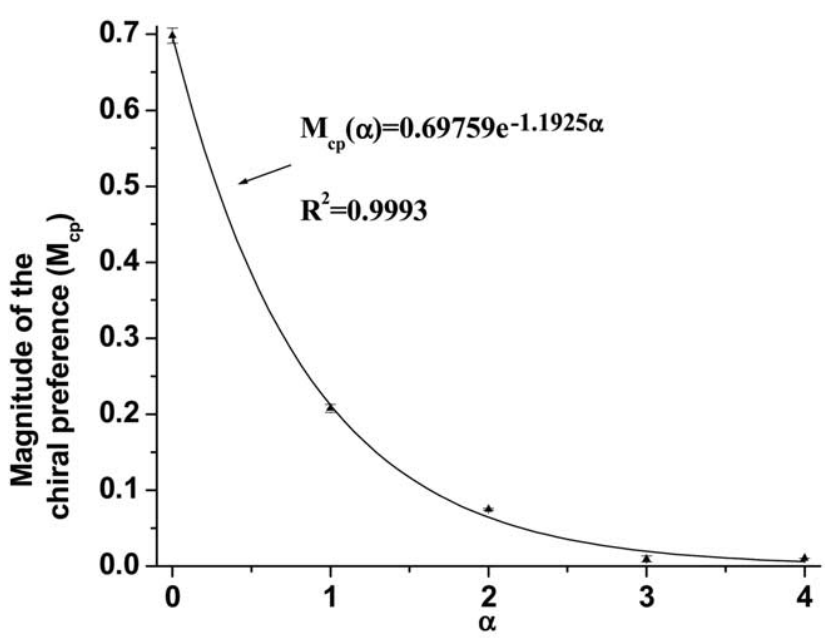

Figure 3. Magnitude of the chiral selectivity involved in the formation of serine octamers obtained from mass spectra recorded using a Finnigan LCQ instrument. The abscissa, $\alpha$, corresponds to the number of minority isomers in the cluster (e.g., for the octamers 8L:0D and 0L:8D $\alpha=0$; for 7L:1D and 1L:7D $\alpha=1$; and so on through 4L:4D where $\alpha=4$ ). The ordinate corresponds to the normalized chiral preference. Best fit to the data is given by an exponential curve.

In order to quantify the observed chiral effects, the term "magnitude of chiral preference" $\left(\mathrm{M}_{\mathrm{cp}}\right)$ is introduced and defined as

$$
M_{c p}(\alpha)=\frac{P_{o b s}\left(L-\operatorname{Ser}_{8-\alpha} D-\operatorname{Ser}_{\alpha}\right)}{P_{t}\left(L-\operatorname{Ser}_{8-\alpha} D-\operatorname{Ser}_{\alpha}\right)}
$$

where $\mathrm{P}_{\mathrm{obs}}$ and $\mathrm{P}_{\mathrm{t}}$ are the observed and theoretical normalized probabilities of formation of the relevant species, while $\alpha$ corresponds to the minority enantiomer in the cluster. Note that $\alpha$ is an integer between 0 and 4; octamers rich in D-Ser are assumed to be equally favored in racemic solutions (for a similar approach see references $[3,6])$. The theoretical probabilities can be calculated using the binomial function. First, $\mathrm{M}_{\mathrm{cp}}$ values were determined for all five types of substituted octamer species when formed from racemic solutions, i.e., under circumstances in which chiral enrichment should not occur. The data are shown in Figure 3. The chiral preference can be seen to drop exponentially as the number of minority enantiomers in the octamers increases. The exponential function can be used to obtain the corrected probabilities of each of the nine possible types of serine octamers, which are defined by eq 2 :

$$
\begin{aligned}
P_{M c p}\left(L-\operatorname{Ser}_{8-x}, D-\operatorname{Ser}_{x}\right) \\
=\frac{M_{c p}(\alpha) \cdot P_{t}\left(L-\operatorname{Ser}_{8-x}, D-\operatorname{Ser}_{x}\right)}{\sum_{\mathrm{i}=0}^{8}\left[M_{c p}(\alpha) \cdot P_{t}\left(L-\operatorname{Ser}_{8-i}, D-\operatorname{Ser}_{i}\right)\right]}
\end{aligned}
$$

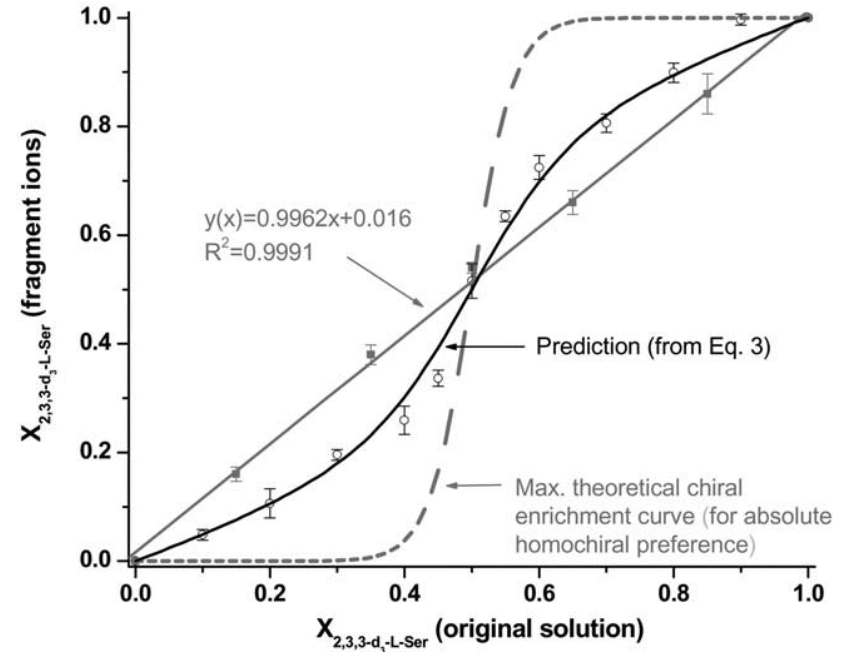

Figure 4. Comparison of experimental and predicted values for $X_{L}$. Black circles correspond to $X_{L}$ calculated from experimental data recorded for the dissociation of heterochiral serine octamers (formed from mixtures of 2,3,3- $\mathrm{d}_{3}$-L-serine/D-serine). The solid black line corresponds to the predicted $X_{L}$ (from eq 3 ), while the gray dashed line is the maximum chiral enrichment curve for serine octamers, that is, for the ideal case of absolute chiral preference (where only formation of D-Ser 8 and L-Ser 8 is allowed). Gray solid line and gray squares experimental data obtained for homochiral serine mixtures $\left(2,3,3-\mathrm{d}_{3}\right.$-L-serine/Lserine), which show a linear trend with slope equal to 1 . This confirms that the effects seen in $\mathrm{L} / \mathrm{D}$ mixtures are indeed related to chirality, ruling out possible isotope effects.

where $\mathrm{M}_{\mathrm{cp}}$ is the magnitude of the homochiral preference and $\alpha$ is the number of minority enantiomers in the octamers (from Figure 3). $\mathrm{P}_{\mathrm{Mcp}}$ is the probability of forming a serine cluster with a strong preference for homochirality (preference factor $\mathrm{M}_{\mathrm{cp}}$ ). Now, from eq 2, it is possible to derive an expression that defines the enantiomeric composition of all clusters; that is the overall amount of each enantiomer that is present in the nine types of serine octamers:

$$
X_{L}=\sum_{i=0}^{8}\left(\frac{8-i}{8}\right) P_{M c p}\left(L-\operatorname{Ser}_{8-\mathrm{i},}, D-\operatorname{Ser}_{i}\right)
$$

where $X_{L}$ is the molar fraction (or probability) of L-serine in all octamers. The value of $X_{L}$ has been obtained experimentally by dissociating octamers formed from solutions of different enantiomeric composition. The experimental results are shown in Figure 4 and compared with the predicted chiral enrichment curve obtained from eq 3 . The good agreement with experiment confirms that the chiral enrichment of serine through formation and dissociation of octamers depends only on the enantiomeric composition of the serine solution and that the chiral preference $\left(\mathrm{M}_{\mathrm{cp}}\right)$ values are intrinsic to octamers of given chiral composition and independent of the chiral composition of the solution. 


\section{Conclusions}

The novel experimental facts shown here support the hypothesis $[2,5,8]$ that serine, through the formation of its homochiral octamers, may have played a key role in homochirogenesis. This role, as argued elsewhere $[2,5$, 8], involved the transmission of chirality to other molecules. The observed increase in enantiomeric excess in cycles of formation and dissociation of serine octamers allows a prebiotic scenario where slight differences in enantiomeric composition might have given rise to octamers of both L- and D-chirality in greatly different proportions. In addition, both enantiomers (monomers) are presumably connected to each other through the uniquely facile chiral interconversion of serine $[8,13$, 14]. Hence, removal of the L-octamers (by adsorption on surfaces such as prebiotic cell membranes [32], crystallization under particular conditions, etc.) could have led to selective accumulated the left-handed serine as part of a cascade of events leading to homochirogenesis, resulting in the L-serine biosphere observed today. Note that this proposal combines the "majority rules" concept [22, 23], well-established in chiral polymerization, with the facile epimerization of serine; note too, though, that the present proposal involves a dynamic, non-equilibrium system which is open. Small numbers of serine molecules may or may not generate octamers which may be removed from the system and this leads to chiral accumulation rather than the predicted result [Selinger and Green, unpublished] of a mixture of homochiral polymers which would occur in a closed system at equilibrium [21].

The recent observation that some serine clusters, especially the 10-mer, undergo an anti-chiral selection process [33] does not remove the possibility that serine clustering, via its octamers, was involved in prebiotic homochirogenesis. Indeed, this possibility is strengthened by the recent observation [34] that the serine octamer is the only significant cluster observed when serine solutions are dropped onto a hot surface (an experiment intended to model early earth conditions in which serine solutions drop onto hot rocks).

The combination of mass spectrometric methodology for creating molecular clusters with ion soft-landing for recovering macroscopic amounts of these materials is a unique aspect of this study and perhaps one with implications for other work on cluster ions.

\section{Acknowledgments}

This work was supported by the National Science Foundation (CHE04-12782 and CHE00-78737) and by the U.S. Department of Energy, Office of Basic Energy Sciences (DE-FG02-94ER14470). SCN thanks Eastman Chemical Co. for fellowship support. The authors thank Hongyian Li, Qizhi Hu, and Robert J. Noll (Purdue University) for the high-resolution experiments and Mark M. Green for valuable discussions.

\section{References}

1. Zhang, D.; Koch, K. J.; Tao, W. A.; Cooks, R. G. Clustering of Amino Acids in the Gas Phase by Electrospray Ionization Mass Spectrometry. Proceedings of the 48th ASMS Conference on Mass Spectrometry and Allied Topics; Long Beach, CA, June 2000.

2. Cooks, R.G.; Zhang, D.; Koch, K. J.; Gozzo, F. C.; Eberlin, M. N. Chiroselective Self-Directed Octamerization of Serine: Implications for Homochirogenesis. Anal. Chem. 2001, 73, 36463655.

3. Hodyss, R.; Julian, R. R.; Beauchamp, J. L. Spontaneous Chiral Separation in Noncovalent Molecular Clusters. Chirality 2001, 13, 703-706.

4. Counterman, A. E.; Clemmer, D. E. Magic Number Clusters of Serine in the Gas Phase. J. Phys. Chem. B 2001, 105, 8092-8096.

5. Koch, K. J.; Gozzo, F. C.; Nanita, S. C.; Takats, Z.; Eberlin, M. N.; Cooks, R. G. Chiral Transmission between Amino Acids: Chirally-Selective Amino Acid Substitution in the Serine Octamer as a Possible Step in Homochirogenesis. Angew. Chem. Int. Ed. 2002, 41, 1721-1724.

6. Julian, R. R.; Hodyss, R.; Kinnear, B.; Jarrold, M.; Beauchamp, J. L. Nanocrystalline Aggregation of Serine Detected by Electrospray Ionization Mass Spectrometry: Origin of the Stable Homochiral Gas Phase Serine Octamer. J. Phys. Chem. B 2002, 106, 1219-1228.

7. Schalley, C. A.; Weis, P. Unusually Stable Magic Number Clusters of Serine with a Surprising Preference for Homochirality. Int. J. Mass Spectrom. 2002, 221, 9-19.

8. Takats, Z.; Nanita, S. C.; Cooks, R. G. Serine Octamer Reactions: Indicators of Prebiotic Relevance. Angew. Chem. Int. Ed. 2003, 42, 3521-3523.

9. Geller, O.; Lifshitz, C. An Electrospray Ionization-Flow Tube Study of H/D Exchange in the Protonated Serine Dimer and Protonated Serine Dipeptide. Int. J. Mass Spectrom. 2003, 227, 77-85.

10. Takats, Z.; Nanita, S. C.; Cooks, R. G.; Schlosser, G.; Vekey, K. Amino Acid Clusters Formed by Sonic Spray Ionization. Anal. Chem. 2003, 75, 1514-1523.

11. Takats, Z.; Nanita, S. C.; Schlosser, G.; Vekey, K.; Cooks, R. G. Atmospheric Pressure Gas-Phase H/D Exchange of Serine Octamers. Anal. Chem. 2003, 75, 6147-6154.

12. Myung, S.; Julian, R. R.; Nanita, S. C.; Cooks, R. G.; Clemmer, D. E. Formation of Nanometer-Scale Serine Clusters by Sonic Spray. J. Phys. Chem. B 2004, 108, 6105-6111.

13. Bada, J. L. In Vivo Racemization in Mammalian Proteins. Methods Enzymol. 1984, 106, 98-115.

14. Nouadje, G.; Nertz, M.; Courderc, F. Study of the Racemization of L-Serine by Cyclodextrin-Modified Micellar Electrokinetic Chromatography and Laser-Induced Fluorescence Detection. J. Chromatogr. A 1995, 716, 331-334.

15. Bonner, W. A.; Lemmon, R. M. Radiolysis, Racemization and the Origin of Molecular Asymmetry in the Biosphere. J. Mol. Evol. 1978, 11, 95-99.

16. Rikken, G. L. J. A.; Raupach, E. Enantioselective Magnetochiral Photochemistry. Nature 2000, 405, 932-935.

17. Hazen, R. M.; Filley, T. R.; Goodfriend, G. A. Selective Adsorption of L- and D-Amino Acids on Calcite: Implications for Biochemical Homochirality. Proc. Natl. Acad. Sci. U.S.A. 2001, 98, 5487-5490.

18. Orme, C. A.; Noy, A.; Wierzbicki, A.; Mcbride, M. T.; Grantham, M.; Teng, H. H.; Dove, P. M.; Deyoreo, J. J. Formation of Chiral Morphologies through Selective Binding of Amino Acids to Calcite Surface Steps. Nature 2001, 411, 775-779.

19. Quack, M.; Stohner, J. Molecular Chirality and the Fundamental Symmetries of Physics: Influence of Parity Violation on 
Rovibrational Frequencies and Thermodynamic Properties. Chirality 2001, 13, 745-753.

20. Cintas, P. Chirality of Living Systems: A Helping Hand from Crystals and Oligopeptides. Angew. Chem. Int. Ed. 2002, 41, 1139-1145.

21. Green, M. M.; Park, J.-W.; Sato, T.; Teramoto, A.; Lifson, S.; Selinger, R. L. B.; Selinger, J. V. The Macromolecular Route to Chiral Amplification. Angew. Chem. Int. Ed. 1999, 38, 31383154.

22. Green, M. M.; Garetz, B. A.; Munoz, B.; Chang, H.; Hoke, S.; Cooks, R. G. Majority Rules in the Copolymerization of Mirror Image Isomers. J. Am. Chem. Soc. 1995, 117, 4181-4182.

23. Li, J.; Schuster, G. B.; Cheon, K.-S.; Green, M. M.; Selinger, J. V. Switching a Helical Polymer Between Mirror Images Using Circularly Polarized Light. J. Am. Chem. Soc. 2000, 122, 26032612.

24. Green, M. M.; Selinger, J. V. Cosmic Chirality. Science 1998, $282,880$.

25. Fenn, J. B.; Mann, M.; Meng, C. K.; Wong, S. F.; Whitehouse, C. M. Electrospray Ionization for Mass-Spectrometry of Large Biomolecules. Science 1989, 246, 64-71.

26. Hirabayashi, A.; Sakairi, M.; Koizumi, H. Sonic Spray Ionization Method for Atmospheric Pressure Ionization Mass Spectrometry. Anal. Chem. 1994, 66, 4557-4559.

27. Miller, S. A.; Luo, H.; Pachuta, S. J.; Cooks, R. G. Soft-Landing of Polyatomic Ions at Fluorinated Self-Assembled Monolayer Surfaces. Science 1997, 275, 1447-1450.
28. Ouyang, Z.; Takats, Z.; Blake, T. A.; Gologan, B.; Guymon, A. J.; Wiseman, J. M.; Oliver, J. C.; Davisson, V. J.; Cooks, R. G. Preparing Protein Microarrays by Soft-Landing of Mass-Selected Ions. Science 2003, 301, 1351-1354.

29. Makarov, A. A. Electrostatic Axially Harmonic Orbital Trapping: A High-Performance Technique of Mass Analysis. Anal. Chem. 2000, 72, 1156-1162.

30. Hardman, M.; Makarov, A. A. Interfacing the Orbitrap Mass Analyzer to an Electrospray Ion Source. Anal. Chem. 2003, 75, 1699-1705.

31. Myung, S.; Lee, Y. J.; Moon, M. H.; Taraszka, J.; Sowell, R.; Koeniger, S.; Hilderbrand, A. E.; Valentine, S. J.; Cherbas, L.; Cherbas, P.; Kaufman, T. C.; Miller, D. F.; Mechref, Y.; Novotny, M. V.; Ewing, M. A.; Sporleder, C. R.; Clemmer, D. E. Development of High-Sensitivity Ion Trap Ion Mobility Spectrometry Time-of-Flight Techniques: A High-Throughput Nano-LC-IMS-TOF Separation of Peptides Arising from a Drosophila Protein Extract. Anal. Chem. 2003, 75, 51375145.

32. Dobson, C. M.; Ellison, G. B.; Tuck, A. F.; Vaida, V. Atmospheric Aerosols as Prebiotic Chemical Reactors. Proc. Natl. Acad. Sci. U.S.A. 2000, 97, 11864-11868.

33. Julian, R. R.; Myung, S.; Clemmer, D. E. Spontaneous AntiResolution in Heterochiral Clusters of Serine. J. Am. Chem. Soc. 2004, 126, 4110-4111.

34. Takats, Z.; Cooks, R. G. Thermal Formation of Serine Octamer Ions. Chem. Commun. 2004, 444-445. 